\title{
Telomerase reverse transcriptase potentially promotes the progression of oral squamous cell carcinoma through induction of epithelial-mesenchymal transition
}

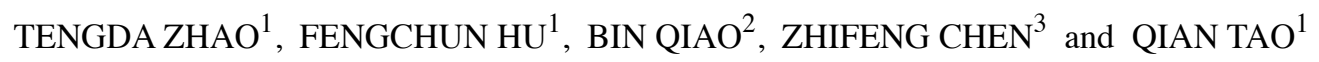 \\ ${ }^{1}$ Guangdong Provincial Key Laboratory of Stomatology, Department of Oral and Maxillofacial Surgery, \\ Guanghua School of Stomatology, Hospital of Stomatology, Sun Yat-sen University, Guangzhou; \\ ${ }^{2}$ Department of Oral and Maxillofacial Surgery, The First Affiliated Hospital of Zhengzhou University, Zhengzhou; \\ ${ }^{3}$ Department of Oral and Maxillofacial Surgery, Nanfang Hospital of Southern Medical University, Guangzhou, P.R. China
}

Received December 28, 2014; Accepted February 18, 2015

DOI: 10.3892/ijo.2015.2927

\begin{abstract}
In recent years, researchers have found the critical role of telomerase in cellular transformation, proliferation, stemness and cell survival. High levels of telomerase reverse transcriptase (TERT) expression and telomerase activation have been reported in most cancer cells. Moreover, overexpression of human TERT (hTERT) is reported to be correlated with advanced invasive stage of the tumor progression and poor prognosis. Epithelial-mesenchymal transition (EMT), characterized by the loss of the cell-cell contact of epithelial cells and the acquisition of migratory and motile properties, is known to be a central mechanism responsible for invasiveness and metastasis of various cancers. Thus, we investigated whether hTERT plays a potential role in the development of EMT. As we expected, our clinical results showed that hTERT is overexpressed in oral epithelial dysplasia (OED) and OSCC tissues and correlates with clinical aggressiveness of oral squamous cell carcinoma (OSCC) patients. We then overexpressed hTERT in primary human oral epithelial cells (HOECS) and found that hTERT has the potential to prolong the lifespan, a process confering the characteristics of EMT by activating the Wnt/ $\beta$-catenin pathway. Our findings provided an explanation for the aggressive nature of human tumors overexpressing hTERT and the possibly mechanism that links hTERT to
\end{abstract}

Correspondence to: Professor Qian Tao, Guangdong Provincial Key Laboratory of Stomatology, Department of Oral and Maxillofacial Surgery, Guanghua School of Stomatology, Hospital of Stomatology, Sun Yat-sen University, Guangzhou, P.R. China

E-mail: taoqian@mail.sysu.edu.cn

Abbreviations: TERT, telomerase reverse transcriptase; OSCC, oral squamous cell carcinoma; HOECS, human oral epithelial cells; EMT, epithelial-mesenchymal transition; OED, oral epithelial dysplasia; NOM, non-cancerous oral mucosa

Key words: telomerase reverse transcriptase, oral squamous cell carcinoma, epithelial-mesenchymal transition, human oral epithelial cells, progression
EMT property, which represents a possible therapeutic target in highly metastatic cancers.

\section{Introduction}

Oral squamous cell carcinoma (OSCC), one of the most common malignant tumors of head and neck, is a leading cause of cancer-related mortality worldwide $(1,2)$. As a multistage process, accumulation of numerous genetic alterations may be involved in the development and progression of OSCC over a long period of time. However, these precise changes that are responsible for OSCC progression are still largely unknown.

Telomerase is an enzyme best known for its role in telomere maintenance (3). Somatic cells lacking telomerase activity lose telomere repeats during cell division, eventually resulting in senescence and apoptosis. Telomerase reverse transcriptase (TERT), as the catalytic subunit of telomerase, has the capacity to limit the activity of telomerase to avoid the erosion of telomere. In recent years, high levels of TERT expression and telomerase activation have been reported in most cancer cells, germ cells and embryonic stem cells, it is well established that hTERT overexpression is a critical step for transformed cells to trigger infinite proliferation during oncogenesis, and that introduction of hTERT into fibroblasts and some epithelial cells confers unlimited replicative potential (4-6). The telomere length-independent effect has also been found for telomerase in cellular transformation (7), proliferation (8), stemness (9) and cell survival (10). Moreover, overexpression of hTERT is reported to be correlated with advanced invasive stage of the tumor progression and poor prognosis (11-14).

Epithelial-mesenchymal transition (EMT), characterized by the loss of the cell-cell contacts of epithelial cells and the acquisition of migratory and motile properties, is known to be a central mechanism responsible for invasiveness and metastasis of various cancers (15). In addition, a recent study revealed that hTERT had a novel role of promoting EMT in gastric cancer cells (16). However, these commonly used carcinoma cell lines also displayed genomic abnormalities unrelated to the devel- 
opment of OSCC, the precise role of hTERT alone is still urgent to clarify in carcinogenesis and cancer progression. We probed into the action mechanisms by overexpression of hTERT in human normal oral epithelial cells (HOECs). Most importantly, we found that hTERT is sufficient to cause EMT, which consequently activates the GSK- $3 \beta / \beta$-catenin pathway, stabilizes Slug and Twist, ultimately, leading to enhanced mitigation and invasiveness of epithelial cells. Taken together, our findings provided an explanation for the aggressive nature of human tumors overexpressing hTERT and the possibly mechanism that links hTERT to EMT.

\section{Materials and methods}

Ethics statements. The study protocol was approved by the institutional ethics board of the Hospital of Stomatology, Sun Yat-sen University, Guangzhou, China. The participants provided their written informed consent before entry into this study. It is recognized that the rights and the welfare of the subjects are adequately protected; the potential risks are outweighed by potential benefits.

Clinical samples and clinical staging system. Paraffinembedded 37 OSCC and 15 oral epithelial dysplasia (OED) samples were obtained from the archives of the Department of Pathology of the Hospital of Stomatology, Sun Yat-sen University, Guangzhou, China. Of the 37 OSCC samples, there were $32(86 \%)$ well- and $5(14 \%)$ moderately differentiated OSCCs, 10 matched adjacent non-cancerous oral mucosa (NOM) were obtained from the above-mentioned patients. All of the samples were treated by surgical excision. For the use of these clinical materials for research purposes, prior patient consent and approval from the Institute Research Ethics Committee was obtained. The disease stages of all the patients were classified according to Union for International Cancer Control (UICC 2002).

Cell culture. Primary cultures of HOECS were established as described previously (17). Briefly, fresh biopsies were obtained from a healthy human adult undergoing third molar extraction. Following rinsing tissues with phosphate-buffered saline (PBS) without calcium and magnesium (containing $1 \%$ penicillin/streptomycin) three times; $0.3-\mathrm{cm}^{2}$ pieces of tissue were incubated in $2 \mu \mathrm{g} / \mathrm{ml}$ Dispase solution (Roche, Germany) for $16-18 \mathrm{~h}$ at $4^{\circ} \mathrm{C}$. The epidermis were gently separated from the dermis using sterile forceps and cut into small pieces, then trpsinized for $5 \mathrm{~min}$ at $37^{\circ} \mathrm{C}$ in $0.125 \%$ trypsin/0.01 mM EDTA. After vigorous pipetting, the cell suspension was centrifuged for $5 \mathrm{~min}$ at $1,000 \mathrm{~g}$ and the cell pellet resuspended in keratinocyte serum-free medium (K-SFM, Invitrogen, USA) supplemented with $25 \mu \mathrm{g} / \mathrm{ml}$ bovine pituitary extract and $0.2 \mathrm{ng} / \mathrm{ml}$ epidermal growth factor. The cells grown were characterized with an anti-cytokeratin antibody (pan ZM-0069, ZSGB-BIO, China). Human oral squamous cell carcinoma Cal27 cells (purchased from ATCC, USA) were maintained in DMEM supplemented with $10 \%$ fetal bovine serum.

Lentiviral vector constructs and siRNA synthesis. Full-length hTERT was amplified from HOEC cDNA and cloned under the control of a CMV promoter into the pEZ-Lv201 lentiviral backbone (Gene Copoeia, Guangzhou, China), containing an eGFP and puromycin-sequence as reporter and selective marker separately. Lentiviruses were generated by transient transfection from HEK 293 T cells. Chemically-modified pre-designed siRNAs were purchased from Invitrogen (Carlsbad, CA, USA). The hTERT-specific RNAi target sequences: hTERT siRNA \#1 (4617): 5-GGUCUUUCU UUUAUGUCACtt-3; hTERT siRNA \#2 (42863): 5-GGUGUC CUGCCUGAAGGACtt-3; hTERT siRNA\#3 (263232): 5-CCA AGCACUU CCUCUACUCtt-3.

Establishment of hTERT-overexpressing HOECs with lentiviral infection. The hTERT gene was introduced into HOEC cells by infecting cells with a lentiviral vector pCMV-hTERT. Control cells were infected with the empty lentiviral vector pCMV. Infected cells were subcultured in K-SFM and subjected to drug selection $(0.5 \mu \mathrm{g} / \mathrm{ml}$ puromycin) for 3-5 days. Cells were serially passaged to determine the replicative lifespan as described (18).

Immunohistochemical analysis. Immunohistochemistry was done to examine hTERT expression in 37 human OSCC tissue specimens, 15 human OED tissue specimens and 10 non-OSCC tissue specimens, hTERT was detected using a rabbit polyclonal antibody against hTERT (Santa Cruz, USA). Briefly, a paraffin section of the tissue from the patient was deparaffinized with xylene, rehydrated and treated with $3 \%$ hydrogen peroxide in methanol to quench the endogenous peroxidase activity. Antigenic retrieval was then processed by submerging the sample in citrate buffer $(\mathrm{pH}$ 6.0) and microwaving followed by incubation with $1 \%$ goat serum albumin to block the nonspecific binding. The sections were then stained with anti-hTERT antibody (1:25) overnight at $4^{\circ} \mathrm{C}$. After washing, the tissue sections were incubated with the goat anti-rabbit/mouse secondary antibody (Gene Tech, Shanghai Co. Ltd., China), then immersed in 3-amino9 -ethyl carbazole (DAB) and counterstained with $10 \%$ Mayer's hematoxylin, dehydrated, and mounted in crystal mount. The degree of immunohistochemical staining of formalin-fixed, paraffin-embedded sections was evaluated by two independent observers, and the mean cytoplasmic and nuclear hTERT staining intensity (SI; 0 , no staining; 1 , weak; 2, moderate; 3 , strong), labeling indices (LIs, defined as the percentage of positive cells in total cells), and mean labeling scores (LSs, defined as LIxSI) in OSCC, OED and NOM samples were calculated and compared among groups.

Confocal immunofluorescence microscopy. Cells were seeded onto the glass slides for $24 \mathrm{~h}$, washed with PBS, fixed in $4 \%$ paraformaldehyde for $20 \mathrm{~min}$ and permeabilized with $0.3 \%$ Triton X-100 for 10 min. After blocking with BSA, cells were stained with the following primary antibodies: rabbit anti-E-cadherin, rabbit anti-vimentin and rabbit anti$\beta$-catenin (1:100, Cell Signaling Technology, MA, USA). The secondary antibody was goat anti-rabbit Alexa Fluor 549 (Earth, USA). Nuclear staining was observed using DAPI. Immunofluorescence was detected by fluorescence microscopy (Olympus, Tokyo, Japan). 
Migration assay. Cells infected with pCMV vector, hTERT was EGF starved for $24 \mathrm{~h}$, then $5 \times 10^{4}$ cells were seeded in medium deprived of EGF into the upper chamber of a polycarbonate transwell filter chamber coated with Matrigel (BD Pharmingen), whereas medium containing EGF was added to the lower chamber. After incubating for $24 \mathrm{~h}$, cells inside the chamber were removed with cotton swabs and migratory cells on the lower membrane surface were fixed in $1 \%$ paraformaldehyde, stained with crystal violet and counted (10 random x100 fields per well). Cell counts are expressed as the mean number of cells per field of view. Three independent experiments were performed and the data are presented as the mean \pm SD.

Wound healing assay. Cells infected with pCMV vectorhTERT were seeded in six-well plates and grown under permissive conditions until reaching $90 \%$ confluence. After starving the cells for $24 \mathrm{~h}$ in medium without EGF, the confluent cell monolayer was lightly and quickly scratched with a pipette tip to create a linear wound, and the debris was removed. Wounds were observed and photographed at various times as indicated in the figure legends. Wound size was measured randomly at five sites perpendicular to the wound. Each experiment was repeated at least three times.

Western blot analyses. Total cellular proteins were extracted with RIPA lysis buffer. BCA method was used for protein quantification. The aliquots were separated on SDS-PAGE $(10 \%)$ and transferred to a nitrocellulose membrane. The following primary antibodies were used: rabbit anti-hTERT (1:1,000, Epitomics, MA, USA); mouse anti-Slug (1:2,000), mouse anti-Twist1 (1:250) (Abcam, Cambridge, MA, USA); mouse anti-p53, rabbit anti-E-cadherin, rabbit anti-vimentin, rabbit anti- $\beta$-catenin, rabbit anti-p-GSK3 $\beta$, rabbit antiGSK3 $\alpha / \beta$ (1:1,000, Cell Signaling Technology); anti-GAPDH (1:2,000, KangChen Bio-tech, Shanghai, China). The proteinantibody complexes were detected by horseradish-conjugated secondary antibodies followed by the enhanced chemiluminescence (ECL) western blotting detection reagents (Pierce, Rockford, IL, USA). Quantification of bands was performed using the Gel-Pro Analyzer.

Statistical analysis. The mean hTERT labeling score (LS) for OSCC, OED, and NOM samples was compared among the three groups by analysis of variance (ANOVA) by using the SPSS 10.0 software package. The correlation between cytoplasmic or nuclear hTERT LSs in OSCCs and clinicopathological characteristics of OSCC patients was analyzed by Student's t-test. $\mathrm{P}<0.05$ was considered statistically significant.

\section{Results}

hTERT is overexpressed in OED and OSCC tissues and correlates with clinical aggressiveness of OSCC patients. To assess the potential role of hTERT in OSCC transformation, immunohistochemical staining was performed to detect whether hTERT is overexpressed in OED and OSCC tissues. As expected, we found that the expression of hTERT increases from NOM to OED and OSCC. In NOE, weak cytoplasmic and moderate nuclear hTERT staining was shown in nearly all
Table I. The mean hTERT LS in NOM, OED and OSCC samples.

\begin{tabular}{lcc}
\hline Groups & $\begin{array}{c}\text { Mean cytoplasmic } \\
\text { hTERT LS } \pm \text { SD }(\%)\end{array}$ & $\begin{array}{c}\text { Mean nuclear } \\
\text { hTERT LS } \pm \text { SD }(\%)\end{array}$ \\
\hline NOM & $53.80 \pm 8.677$ & $123.80 \pm 14.771$ \\
OED & $141.40 \pm 16.115$ & $217.60 \pm 35.944$ \\
OSCC & $187.65 \pm 10.698$ & $215.03 \pm 42.502$
\end{tabular}

A significant difference in the mean cytoplasmic hTERT LS was found among NOM, OED and OSCC groups ( $\mathrm{P}=0.000)$. LS, labeling scores; NOM, non-cancerous oral mucosa; OED, oral epithelial dysplasia; OSCC, oral squamous cell carcinoma.

epithelial cells, the cytoplasmic and nuclear staining intensity of basal layers was much denser than that of upper epithelial cells; in OED, moderate cytoplasmic and strong nuclear hTERT staining was exhibited in nearly all epithelial cells, basal layers with mitotic figures showed a stronger staining intensity than other cells without mitotic figures, and in OSCC, moderate to strong cytoplasmic and nuclear hTERT staining was exhibited in nearly all epithelial cells. Combined with pathological analysis, these results suggested that the distribution of activated hTERT in epithelium at different stages may represent a biomarker of the degree of malignant transformation in oral carcinogenesis. Representative microphotographs of hTERT staining for NOM, OED and OSCC are shown in Fig. 1A. The mean cytoplasmic hTERT LS increased significantly

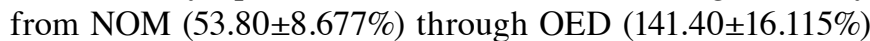
to OSCC samples $(187.65 \pm 10.698 \%)$ (Table I). A significant difference in the mean cytoplasmic hTERT LS was found between OSCC and OED ( $\mathrm{P}=0.000)$ or NOM samples $(\mathrm{P}=0.000)$. The mean nuclear hTERT LS increased from NOM $(123.80 \pm 14.771 \%)$ to OED $(217.60 \pm 35.944 \%)$, and then decreased to OSCC samples $(215.03 \pm 42.502 \%)$, there was no significant difference in the mean nuclear hTERT LS among the 3 groups.

We further analyzed the correlation between cytoplasmic and nuclear hTERT LS, respectively, in OSCC samples and clinical characteristics of OSCC patients (Fig. 1B). There was no difference correlation between the cytoplasmic or nuclear expression of hTERT and age, gender, location and N classification. However, there was a significant difference of the cytoplasmic hTERT expression from well to moderatelydifferentiated OSCCs according to histology classification $(\mathrm{P}=0.003)$. In addition, OSCCS with larger tumor sizes (T3 and T4) had a significantly higher mean nuclear hTERT LS than OSCCs with smaller tumor sizes (T1 and T2, $\mathrm{P}=0.005$ ) (Table II). Thus, our findings indicated that hTERT is correlated with clinical aggressiveness of OSCC patients.

Establishment of hTERT-overexpressing human OEC cell line. To exclude the involvement of other mutant genes, and further investigate the role of hTERT alone in the development of oral squamous cell carcinoma. We firstly established hTERT-overexpressing cells in primary HOEC, by which we investigated its action mechanism. We observed that the primary adherent cells were flat, and polygonal in low 
A

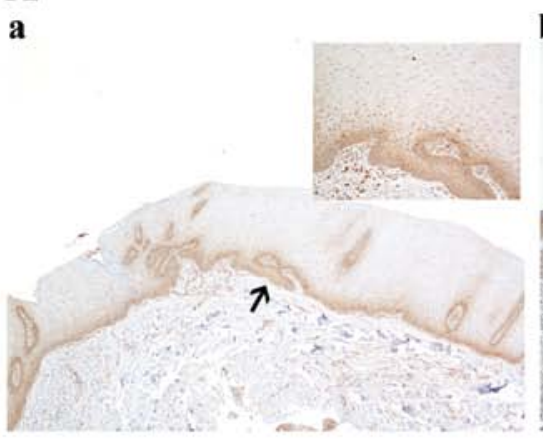

B b

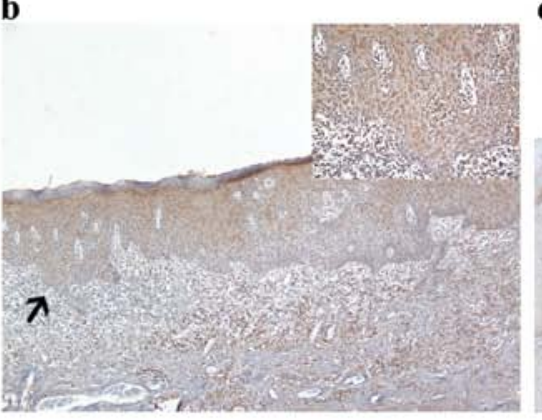

c

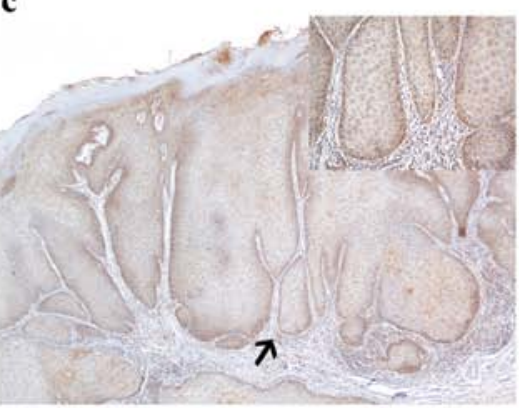

b

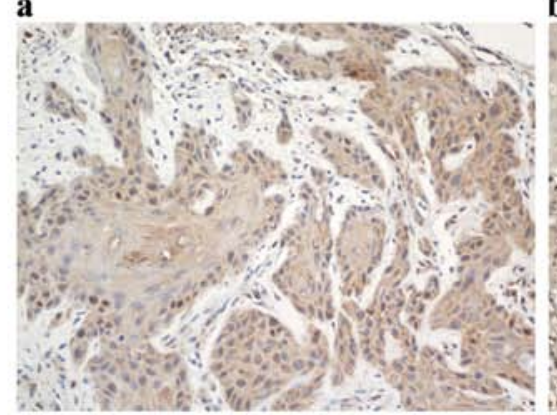

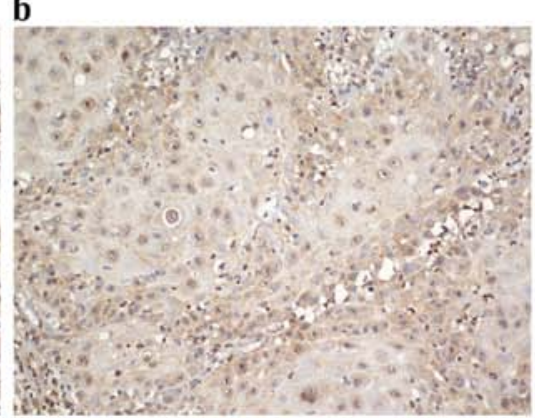

Figure 1. Immunohistochemical staining for hTERT in oral epithelium (A) and tumor nests (B). (A) (a) Normal oral epithelium showed weak cytoplasmic hTERT staining and moderate nuclear hTERT staining in nearly all epithelial cells. The cytoplasmic and nuclear staining intensity of basal layers (arrow, x200) was much denser than that of upper epithelial cells. (b) Oral epithelial dysplasia exhibited moderate cytoplasmic hTERT staining and strong nuclear hTERT staining in nearly all epithelial cells. Basal layers with mitotic figures (arrow, x200) showed a stronger staining intensity than other cells without mitotic figures. (c) Oral squamous cell carcinoma exhibited moderate to strong cytoplasmic and nuclear hTERT staining in nearly all epithelial cells (arrow, $\mathrm{x} 200$ ). (B) ( $\mathrm{a}$ and b) Cells from central parts of the nests from well- to moderately-differentiated squamous tumor showed moderate to strong cytoplasmic and nuclear hTERT staining and overexpression of hTERT was associated with tumor differentiation [(A) (a, b and c) x40 magnification; (B) (a and b) x200 magnification)].

Table II. Correlation between cytoplasmic or nuclear hTERT LS in OSCC samples and clinical characteristics of OSCC patients.

\begin{tabular}{ccc}
\hline Characteristics & Mean cytoplasmic & Pean nuclear \\
hTERT LS \pm SD $(\%)$ & P-value & hTERT LS \pm SD $(\%)$
\end{tabular}

Age

$$
<50(\mathrm{n}=11)
$$$$
\geq 50(n=26)
$$

$1.8636 \pm 0.11944$

$1.8819 \pm 0.10331$

0.703

Gender

Male $(n=24)$

$1.8867 \pm 0.11761$

$1.8577 \pm 0.08506$

0.083

$1.8781 \pm 0.10221$

$1.8744 \pm 0.11633$

0.245

$1.8621 \pm 0.10016$

$1.9288 \pm 0.12147$

0.584

$1.8612 \pm 0.10025$

$1.9127 \pm 0.11841$

0.375

$1.8500 \pm 0.08861$

$2.0460 \pm 0.02510$

Histology of OSCC

WD OSCC $(n=32)$

$\operatorname{MD} \operatorname{OSCC}(n=5)$
$1.9909 \pm 0.38524$

$2.2177 \pm 0.42995$

0.537

$2.2142 \pm 0.43902$

$2.0323 \pm 0.38635$

$2.1052 \pm 0.39878$

$2.2094 \pm 0.46362$

0.283

$2.0607 \pm 0.42705$

$2.4750 \pm 0.21394$

0.005

$2.0585 \pm 0.40896$

$2.3673 \pm 0.39810$

0.463

$2.1341 \pm 0.43108$

$2.2540 \pm 0.41956$

0.415

Comparison between groups was performed by Student's t-test. LS, labeling scores; WD, well-differentiated; MD, moderately differentiated. 


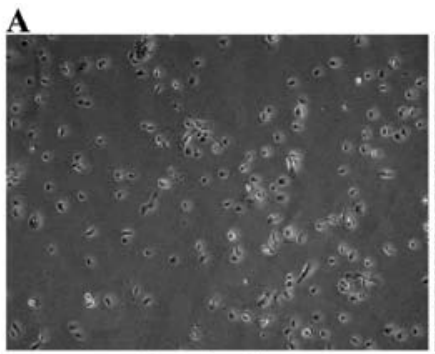

Low density

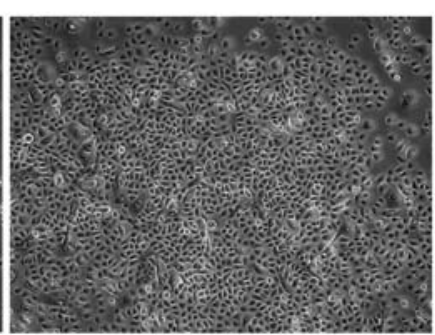

High density
B

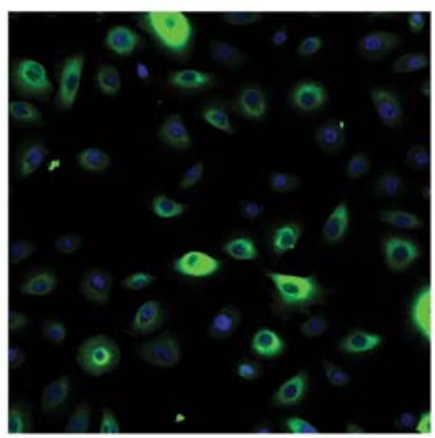

Figure 2. Establishment and identification of a HOEC cell line. (A) The primary culture of HOECs showed polygonal morphology and a flagstone arrangement at different cell densities. (B) Identification was by confoca microscopy, green indicates positive staining of cytokeratin in the HOEC cytoplasm, while the blue signal signifies nuclear DNA staining with DAPI. Scale bar, $100 \mu \mathrm{m}$. density, then grew attached to form a typical cobblestone-like morphology, which is a characteristic of epithelial cells grown in culture (Fig. 2A). To identify the origin of these cells, the expression of pan-cytokeratin (AE1/AE3) was detected. The result showed green-positive staining for cytokeratin in the cytoplasm of each cell, indicating that they were of epithelial origin (Fig. 2B).

Next, we overexpressed hTERT in HOECs by infecting with a lentiviral vector expressing hTERT or control vector at the 12th PDS of the best activity of cellular proliferation. After puromycin selection, the eGFP fluorescence of stable infection with hTERT cells was visualized under a fluorescent microscope and overexpression of hTERT was confirmed by western blot analysis (Fig. 3A and B).

Overexpression of hTERT prolongs the lifespan and downregulates p53 expression of HOECs. It is known that the primary keratinocytes, as terminally differentiated cells, have a finite lifespan and quickly undergo obvious senescence. As expected, HOECs infected with the control lentivirus proliferated for a limited number of passages (nearly 20 population doublings after selection) and then underwent senescence, while hTERT-overexpressing cells had an extended lifespan and were able to proliferate for over 150 population doublings (Fig. 3C). We assessed the expression of p53, a pro-apoptosis molecular downstream of hTERT, in control and hTERT-

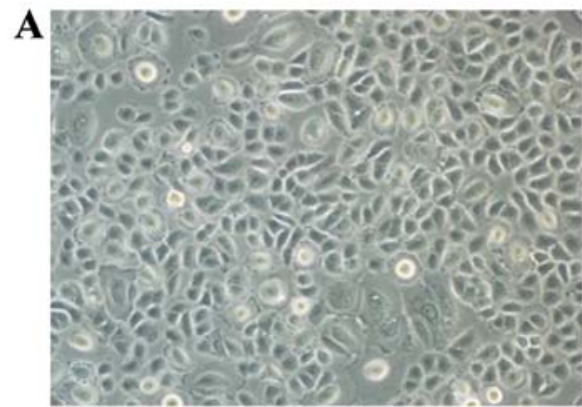

Bright field

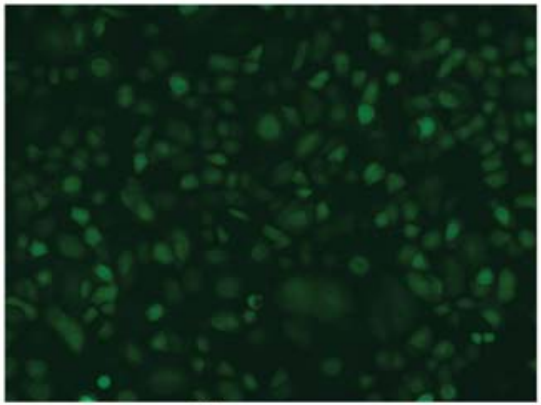

Fluorescence
B

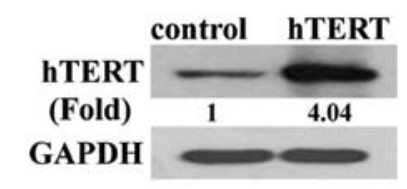

D

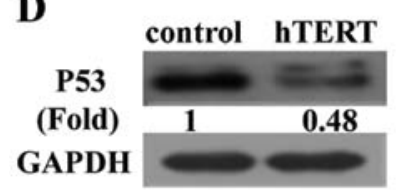

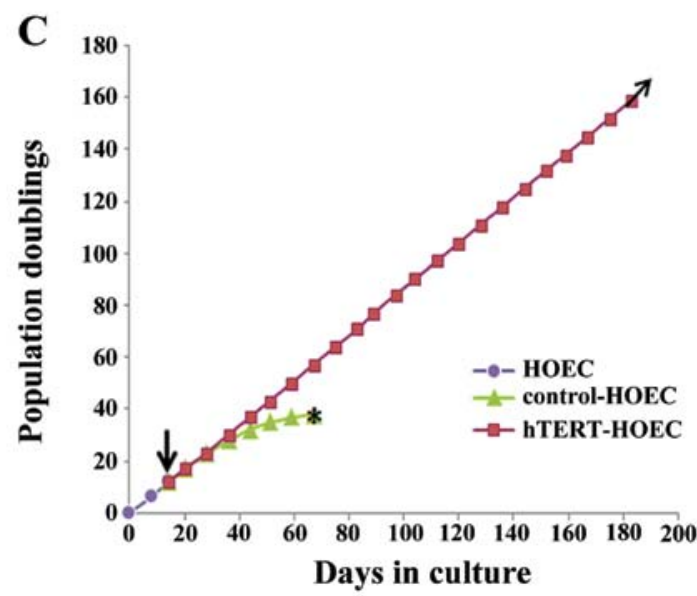

Figure 3. Overexpression of hTERT prolongs the lifespan and downregulates p53 expression of HOECs primary HOECs. (A) The expression of eGFP reporter of hTERT-HOECs was checked with a fluorescence microscope 3 days post-selection. (B) Total cell lysates of control (pCMV) and hTERT-overexpressing (pCMV-hTERT) cells were analyzed by western blot analysis using anti-hTERT antibodies. Anti-GAPDH was used as loading control. (C) Population doublings (PDS) was achieved by hTERT and control lentiviral-infected HOECs at an early stage of their respective lifespans. Downward arrow indicates the time of transduction. (D) Repression of P53 pathway in hTERT-overexpressing HOECs (at 35 PDS) and pCMV vector control cells were analyzed by western blot analysis using antibodies against P53. Anti-GAPDH was used as a loading control. 


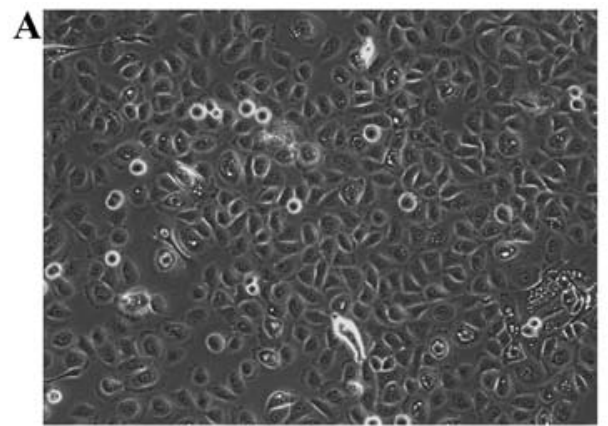

Epithelial morphology

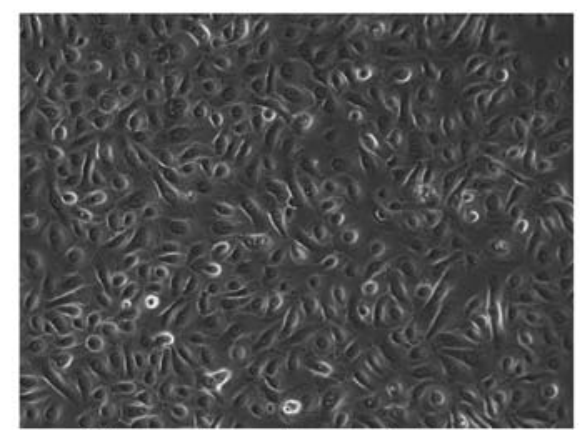

Mesenchymal morphology

C
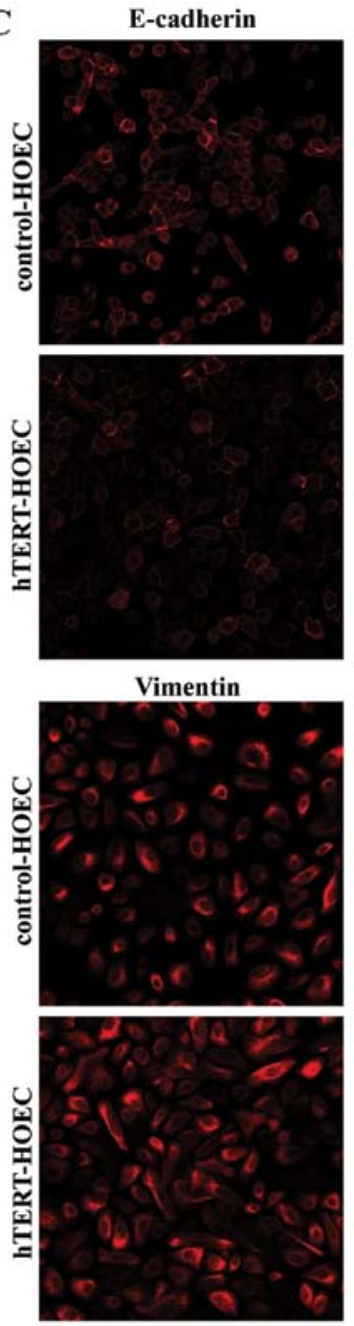

DAPI
B
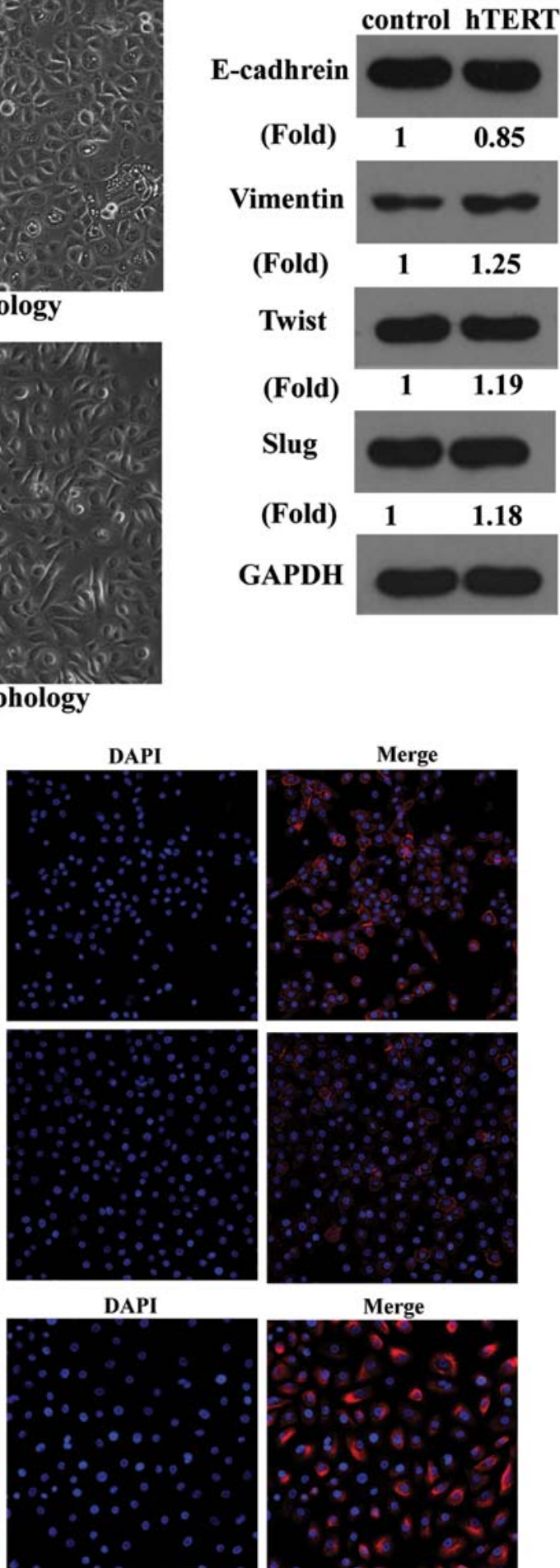

Merge
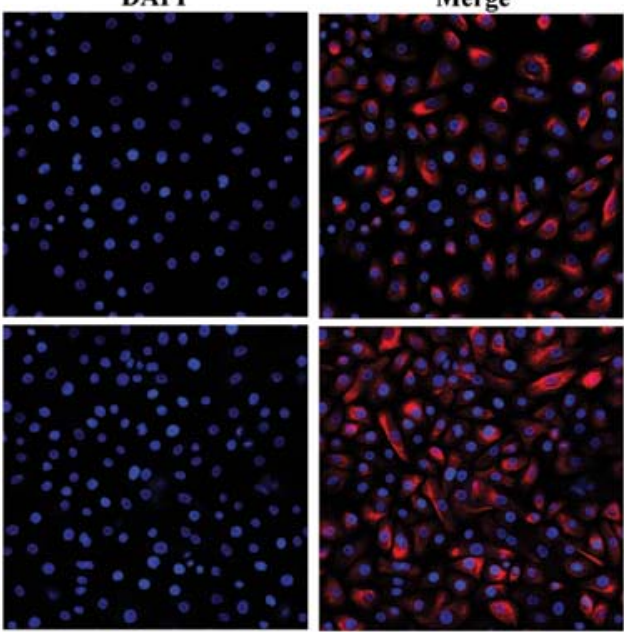

Figure 4. Overexpression of hTERT promotes EMT-like molecular alterations in HOECs. (A) The cellular morphology of HOECS expressing either the control vector pCMV or pCMV/hTERT was shown by phase contrast microscopy. Scale bar, $100 \mu \mathrm{m}$. (B) Expression of the epithelial proteins E-cadherin, the mesenchymal proteins vimentin and EMT transcription factors Slug and Twist1 in HOECs expressing control vector pCMV or pCMV/hTERT were detected by western blot analysis. GAPDH was used as a loading control. (C) HOECs expressing control vector pCMV or pCMV/hTERT was replated on K-SFMprecoated coverslips. After an additional $24 \mathrm{~h}$, cells were stained for E-cadherin, vimentin, and analyzed by confocal microscopy. The red signal represents staining for the corresponding protein, while the blue signal signifies nuclear DNA staining with DAPI. Scale bar, $100 \mu \mathrm{m}$. 
A
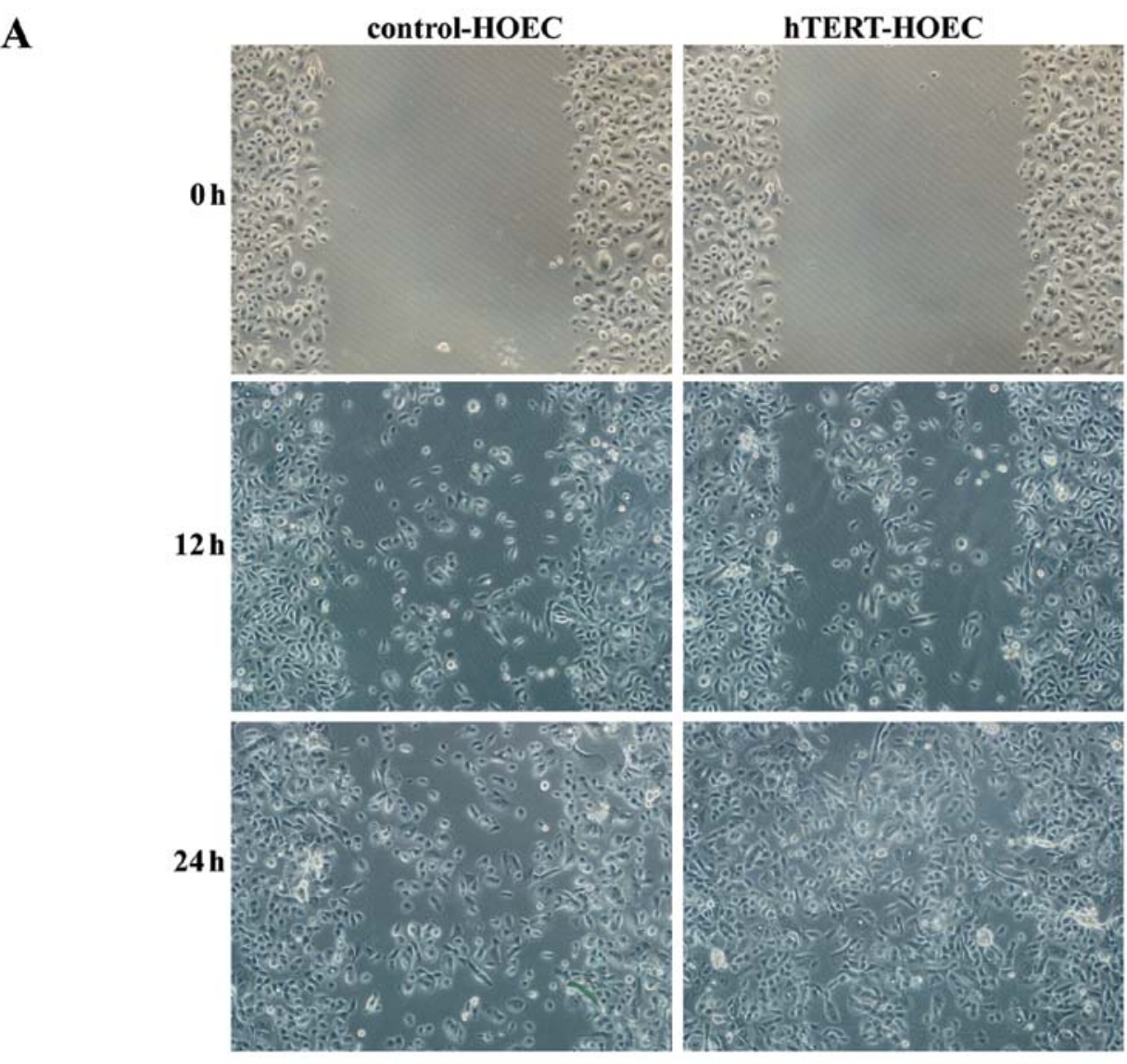

B

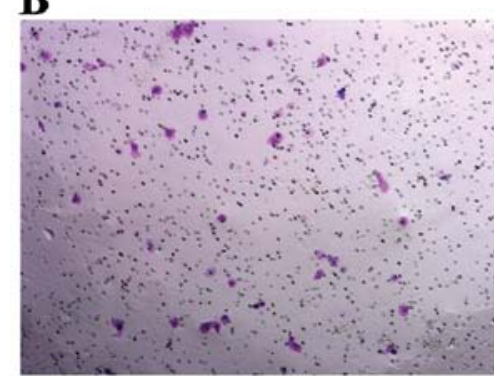

control-HOEC

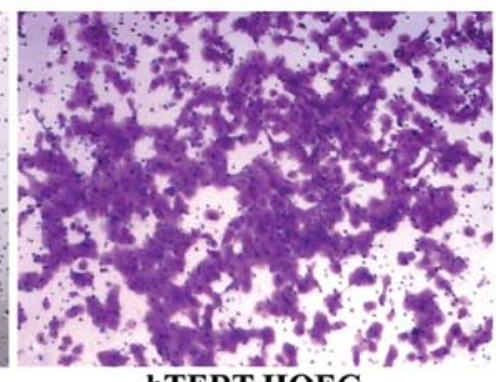

hTERT-HOEC

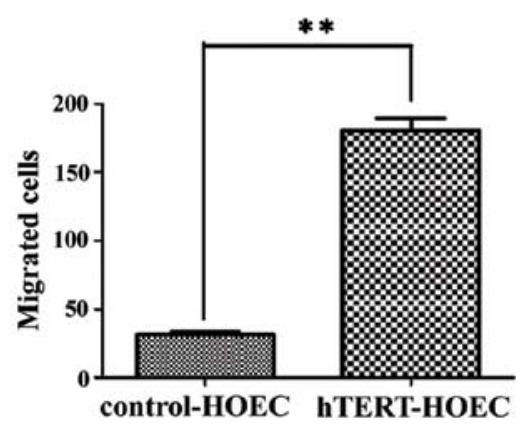

Figure 5. Overexpression of the hTERT enhances cell motility and invasiveness in HOECs. (A) A wound was introduced in subconfluent cultures of hTERT-overexpressing or control vector cells. The rate of wound closure was monitored at different times as indicated. A representative image from three independent experiments is shown. Scale bar, $100 \mu \mathrm{m}$. (B) The invasive properties of the cells were analyzed in by an invasion assay using a Matrigel-coated Boyden chamber. Migrated cells were plotted as the average number of cells per field of view from 3 different experiments, as described in Materials and methods. Scale bar, $100 \mu \mathrm{m}$. Error bars represent SEM. ${ }^{* *} \mathrm{P}<0.01$.

overexpressing cells by western blot analysis. The result showed that the expression of p53 was significantly reduced in hTERT-overexpressing cells (Fig. 3D).

Ectopic expression of hTERT induces a mesenchymal phenotype and enhances the invasiveness of HOECs. During the process of several serial passages after cell transfection, a slight morphological change was observed in hTERToverexpressing HOECs at nearly 20 PDS, in which the typical cobblestone-like appearance of normal epithelium was replaced by a spindle-like morphology (Fig. 4A), which suggested that hTERT-HOECs might have undergone EMT. In order to determine whether hTERT promotes EMT, we analyzed the cells with a panel of epithelial and mesenchymal markers. As shown in Fig. 4B, there was a slight reduction in E-cadherin expression, whereas vimentin expression was enforced, the upregulated expression of EMT transcription factors Slug and Twist1 were also readily observed in hTERT-HOECs. The EMT phenotype was further confirmed by immunofluorescent staining (Fig. 4C). Because the EMT program activation contributes to cancer cell mobility and consequent invasion, we next examined the effect of hTERT on cell motility and invasive potential using wound healing and transwell assay coated with Matrigel. As shown in Fig. 5A, the scratch wound-healing assay also indicated that HOECs overexpressing hTERT exhibited significantly enhanced 
A
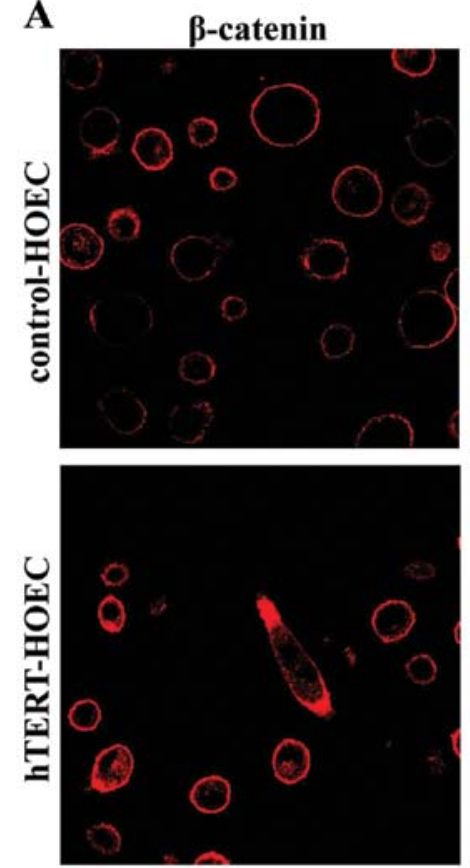
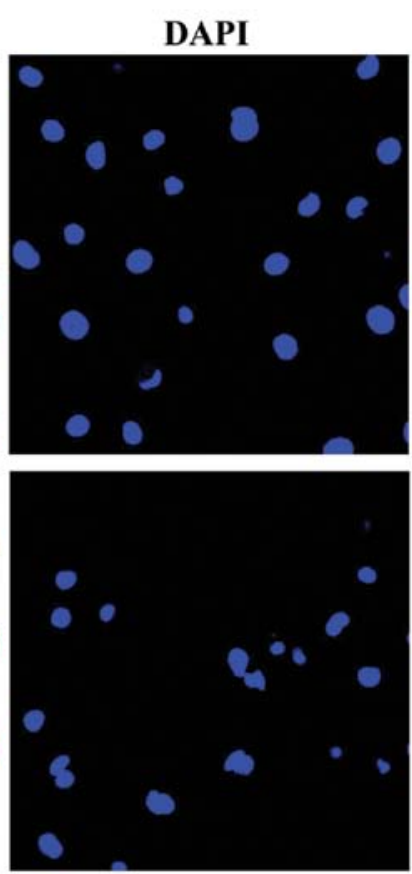
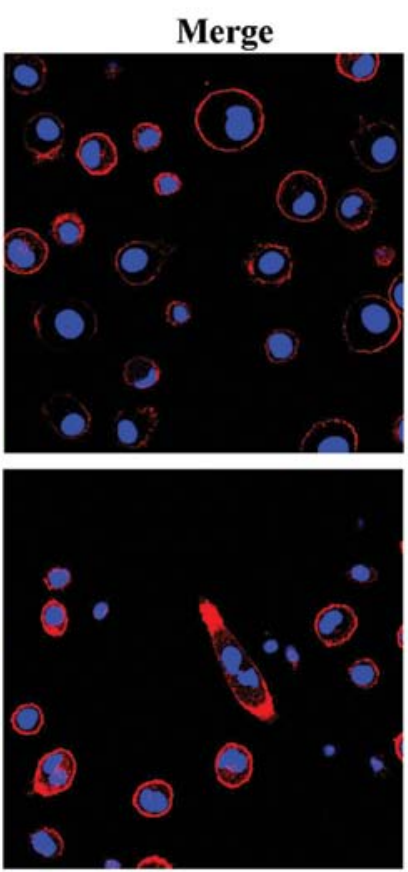

B

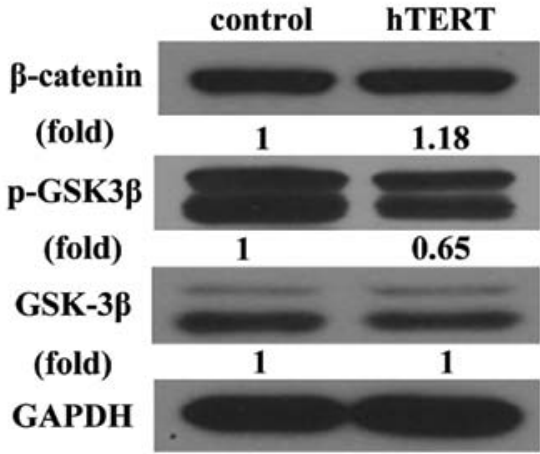

Figure 6. Upregulation of hTERT activates the Wnt/ $\beta$-catenin pathway and induces cytoplasmic accumulation of $\beta$-catenin. (A) Expression of the $\beta$-catenin, $\mathrm{p}-\mathrm{GSK} 3 \beta$ and GSK3$\beta$ proteins in HOEC cells expressing control vector pCMV or pCMV/hTERT were detected by western blot analysis GAPDH was used as a loading control. (B) Subcellular location of $\beta$-catenin in HOECs expressing control vector $\mathrm{pCMV}$ or $\mathrm{pCMV/hTERT} \mathrm{was} \mathrm{examined}$ by confocal microscopy. The red signal represents staining for the $\beta$-catenin protein, while the blue signal signifies nuclear DNA staining with DAPI. Scale bar, $100 \mu \mathrm{m}$.

mobility compared with vector control cells. Moreover, hTERT-HOECs displayed significantly increased invasiveness in comparison with vector control cells (Fig. 5B).

Upregulation of hTERT activates the Wnt/ $\beta$-catenin pathway and induces cytoplasmic accumulation of $\beta$-catenin. Aberrant activation of the Wnt/ $\beta$-catenin has a critical role in promoting EMT during human tumorigenesis (19). Furthermore, it is established that TERT is the direct modulator of the wnt/ $\beta$-catenin pathway (20). We thus asked whether EMT promoted by hTERT is associated with activation of $\beta$-catenin in HOECs. As shown in Fig. 6A, overexpression of hTERT decreased the phosphorylation of GSK-3 $\beta$ and contributed to slight upregulation of $\beta$-catenin expression, thus we further examined the subcellular location of $\beta$-catenin. Compared with control vector HOECS, the cytoplasmic accumulation of $\beta$-catenin increased, parts of which entered into nuclear in hTERT-overexpressed HOECS (Fig. 6B), suggesting that upregulation of hTERT activates the GSK-3 $\beta / \beta$-catenin pathway, stabilizes Slug and Twist, and upregulates vimentin, ultimately leading to enhanced migration and invasiveness in hTERT-HOECS.
Silencing endogenous hTERT represses mesenchymal phenotype and inhibits the Wnt/ $\beta$-catenin pathway in OSCC cells. To test and verify the impact of hTERT on EMT, we knocked down endogenous hTERT in oral squamous cancer cell line Cal27 using hTERT-specific small interfering RNA (siRNA) and examined the resulting phenotype. These three siRNAs all specifically knocked down endogenous hTERT protein in the Cal27 cell line, siRNA \#3 was more efficient compared with the other siRNAs, and was therefore chosen for subsequent studies (Fig. 7A). As shown in Fig. 7B, silencing endogenous hTERT in Cal27 cells led to enhanced expression of epithelial markers E-cadherin and concomitant decreased expression of the mesenchymal marker vimentin. Moreover, the expression of EMT transcription factors Slug and Twist1 were also markedly downregulated. We next examined whether depletion of hTERT could inhibit the Wnt/ $\beta$ catenin pathway. As shown in Fig. 7C, depletion of hTERT increased the phosphorylation of GSK-3 $\beta$ and markedly downregulated $\beta$-catenin expression, which suggested that depletion of endogenous hTERT inhibits the Wnt/ $\beta$-catenin pathway and leads to reverse EMT in OSCC cells. 
A

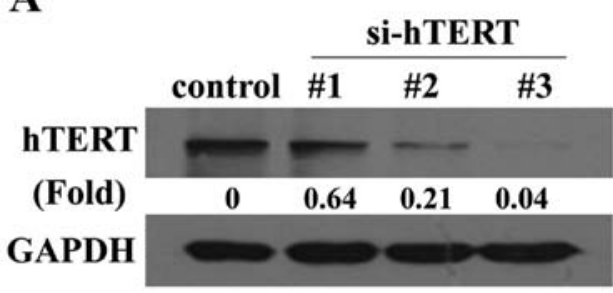

C

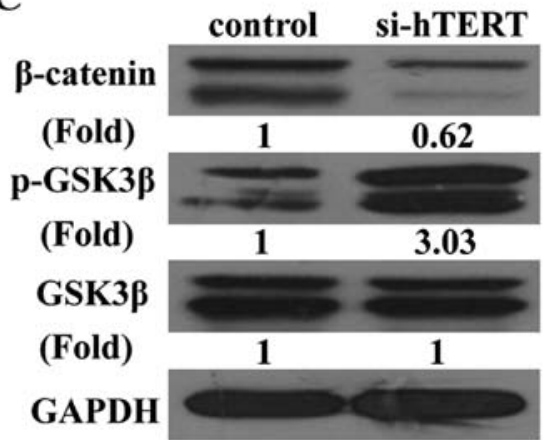

B

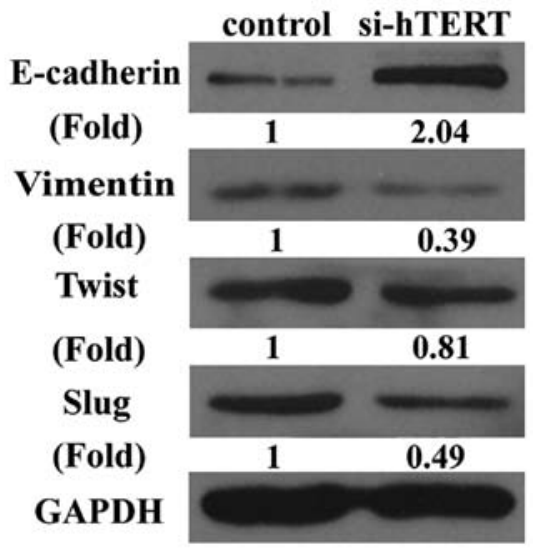

Figure 7. Silencing endogenous hTERT expression represses mesenchymal phenotype and inhibits the Wnt/ $\beta$-catenin pathway in Cal27 cells. (A) Expression of hTERT in control and hTERT-silenced Cal27 cells were analyzed by western blot analysis using anti-hTERT antibodies. Anti-GAPDH was used as loading control. (B) Expression of the epithelial protein E-cadherin, the mesenchymal protein vimentin and EMT transcription factors Slug and Twist1 in Cal27 cells was detected by western blot analysis. GAPDH was used as a loading control. (C) Expression of the $\beta$-catenin, p-GSK3 $\beta$ and GSK3 $\beta$ proteins in control and hTERT-silenced Cal27 cells was detected by western blot analysis. Anti-GAPDH was used as a loading control.

\section{Discussion}

OSCC, as the most frequent malignant tumor of the oral cavity, has highly metastatic characteristics (21). Recent observations suggest that EMT is involved in oncogenesis, invasion and metastasis $(22,23)$. Efforts to elucidate the underlying molecular mechanism of these oral malignant transformation processes are therefore warranted in order to develop novel therapeutic approaches. In the present study, we provide evidence that hTERT confers the characteristics of EMT by activating the Wnt/ $\beta$-catenin pathway.

Numerous studies have revealed that the accumulation of multiple genetic alterations over a long period of time may be involved in the development and progression of cancer. Higher levels of expression of hTERT are associated with almost all human malignant tumors but not in benign or normal tissues with the exception of germ cells, stem cells, and activated lymphocytes. Ectopic expression of hTERT has been demonstrated in diseases arising from lung cancer (24), gastric cancer (25), hepatocellular cancer (26), breast cancer (27) and bronchial mucosa of heavy smokers (28). In addition, it has been suggested that hTERT was a prognostic marker for human malignancies $(29,30)$. Our clinical results showed that hTERT is overexpressed in OED and OSCC tissues and correlates with clinical aggressiveness of OSCC patients, which proved its important role in the development of oral carcinomas. Furthermore, the typical distribution of activated hTERT in epithelium might suggest hTERT is a valuable molecular marker to diagnose the early stage of malignant transformation in the development of OSCC.
Over the past years, various OSCC-derived cell lines have been established. However, its specific molecular mechanism of oral carcinoma is far from being fully understood, the main fact is that such cell lines also display genomic abnormalities unrelated to OSCC development. In our study, to overcome the above drawback and to be able to investigate the potential role of hTERT in promoting the development of oral carcinogenesis and progression in a defined genetic elements manner, we first established an hTERT-overexpressing HOECS cell line with a single cellular hTERT gene, which is always ectopically expressed in OSCC tumor. As shown in our study, excluded from other undefined genetic lesions, the hTERT gene could possess the capacity to promote EMT.

EMT is well known for its major role in embryogenesis and development, in which epithelial cells acquire properties reminiscent of those of mesenchymal cells. As an important cancer-related process, EMT is often involved in invasion and metastasis during tumor progression. Herein, we demonstrated that hTERT possesses the capacity to promote the characteristics of EMT in HOECs. In hTERT-HOECs, a slight spindle-like morphology was observed at nearly 20 PDS. Further analysis proved that there was a slight reduction in E-cadherin expression and enforced expression of vimentin, the expression of EMT transcription factors Slug and Twist1 were also upregulated. Moreover, silencing endogenous hTERT led to the reverse trend in Cal27 cells. However, unlike other cancer cell lines, the primary HOECs exhibiting partial mesenchymal phenotype changes require several passages under selection after introduction of hTERT (often after 15 PDS). The degree of change in the EMT transcription factors were less than that in Cal27 cells. Probing deeper into the 
reason, one of the putative explanations for this discrepancy is that this hTERT-overexpressing HOEC cell line might have a stronger self-defense ability to resist these reverse reactions and lack of the involvement of other oncogenes to affect EMT compared with the cancer cells.

Recent reports have shown that multiple effectors including the PI3 and MAP kinases $(31,32)$ and Wnt $(33,34)$, Notch (35) and NF- $\mathrm{B}$ (36) pathways can be activated to be involved in EMT regulation. With respect to the molecular link between TERT and Wnt/ $\beta$-catenin signaling, evidence showed that TERT and $\mathrm{Wnt} / \beta$-catenin pathway activation are bidirectional regulation in embryonic stem (ES) cells and cancer, $\beta$-catenin could regulate TERT expression through the interaction with the pluripotent transcription factor Klf4 (37), while TERT directly interacts with $\beta$-catenin (16) or its target gene BRG1 (20) to activate Wnt/ $\beta$-catenin pathway. In the present study, our results revealed that hTERT promotes the EMT program through activation of $\mathrm{Wnt} / \beta$-catenin pathway, overexpression of hTERT decreased the phosphorylation of GSK-3 $\beta$ and translocated $\beta$-catenin into nucleus, while depletion of hTERT inhibited the GSK-3 $\beta / \beta$-catenin pathway. Thus, hTERT potentially promotes EMT by activation of Wnt signaling, which suggested a unique status in carcinogenesis and progression.

In conclusion, hTERT plays a pivotal role in the development and progression of OSCC. Our results provide an explanation for the aggressive nature of human tumors overexpressing hTERT and the possibly mechanism that links hTERT to EMT property, which may partially by targeting activation of the Wnt/ $\beta$-catenin pathway. Hence, we believe that hTERT can be a therapeutic target for high-metastasis cancers and further uncovering the exact function and molecular mechanism for hTERT overexpression in cancer would provide important insight into understanding tumor progression and metastasis.

\section{Acknowledgements}

This study was supported by the National Natural Science Foundation of China (grant no. 81072227).

\section{References}

1. Bray F, Sankila R, Ferlay J and Parkin DM: Estimates of cancer incidence and mortality in Europe in 1995. Eur J Cancer 38: 99-166, 2002.

2. Parkin DM, Bray F, Ferlay J and Pisani P: Global cancer statistics, 2002. CA Cancer J Clin 55: 74-108, 2005.

3. Liu JP, Chen SM, Cong YS, Nicholls C, Zhou SF, Tao ZZ and Li H: Regulation of telomerase activity by apparently opposing elements. Ageing Res Rev 9: 245-256, 2010.

4. Shay JW and Wright WE: Hallmarks of telomeres in ageing research. J Pathol 211: 114-123, 2007.

5. Kapanadze B, Morris E, Smith E and Trojanowska M: Establishment and characterization of scleroderma fibroblast clonal cell lines by introduction of the hTERT gene. J Cell Mol Med 14: 1156-1165, 2010.

6. Wieser M, Stadler G, Jennings P, Streubel B, Pfaller W, Ambros P, Riedl C, Katinger H, Grillari J and Grillari-Voglauer R: hTERT alone immortalizes epithelial cells of renal proximal tubules without changing their functional characteristics. Am J Physiol Renal Physiol 295: F1365-F1375, 2008.

7. Stewart SA, Hahn WC, O'Connor BF, et al: Telomerase contributes to tumorigenesis by a telomere length-independent mechanism. Proc Natl Acad Sci USA 99: 12606-12611, 2002.
8. Smith LL, Coller HA and Roberts JM: Telomerase modulates expression of growth-controlling genes and enhances cell proliferation. Nat Cell Biol 5: 474-479, 2003.

9. Yang C, Przyborski S, Cooke MJ, Zhang X, Stewart R, Anyfantis G, Atkinson SP, Saretzki G, Armstrong L and Lako M: A key role for telomerase reverse transcriptase unit in modulating human embryonic stem cell proliferation, cell cycle dynamics, and in vitro differentiation. Stem Cells 26: 850-863, 2008.

10. Lee J, Sung YH, Cheong C, Choi YS, Jeon HK, Sun W, Hahn WC, Ishikawa $\mathrm{F}$ and Lee HW: TERT promotes cellular and organismal survival independently of telomerase activity. Oncogene 27: 3754-3760, 2008.

11. Bertorelle R, Briarava M, Rampazzo E, et al: Telomerase is an independent prognostic marker of overall survival in patients with colorectal cancer. Br J Cancer 108: 278-284, 2013.

12. Eid MM, Helmy NA, Omar IM, Mohamed AA, El Sewefy D, Fadel IM and Helal RA: Clinical significance of telomerase genes (hTERC and hTERT) amplification in patients with acute myeloid leukemia. Gulf J Oncol 1: 51-60, 2013.

13. Lötsch D, Ghanim B, Laaber M, Wurm G, Weis S, Lenz S, Webersinke G, Pichler J, Berger W and Spiegl-Kreinecker S: Prognostic significance of telomerase-associated parameters in glioblastoma: Effect of patient age. Neuro-oncol 15: 423-432, 2013.

14. Nakashima A, Murakami Y, Uemura K, Hayashidani Y, Sudo T, Hashimoto Y, Ohge H, Oda M, Sueda T and Hiyama E: Usefulness of human telomerase reverse transcriptase in pancreatic juice as a biomarker of pancreatic malignancy. Pancreas 38 : 527-533, 2009.

15. Tsuji T, Ibaragi S and Hu GF: Epithelial-mesenchymal transition and cell cooperativity in metastasis. Cancer Res 69: 7135-7139, 2009.

16. Liu Z, Li Q, Li K, et al: Telomerase reverse transcriptase promotes epithelial-mesenchymal transition and stem cell-like traits in cancer cells. Oncogene 32: 4203-4213, 2013.

17. Gröger S, Michel J and Meyle J: Establishment and characterization of immortalized human gingival keratinocyte cell lines. J Periodontal Res 43: 604-614, 2008.

18. Rheinwald JG, Hahn WC, Ramsey MR, Wu JY, Guo Z, Tsao H, De Luca M, Catricala C and O'Toole KM: A two-stage, p16 (INK4A)- and p53-dependent keratinocyte senescence mechanism that limits replicative potential independent of telomere status. Mol Cell Biol 22: 5157-5172, 2002.

19. Dissanayake SK, Wade M, Johnson CE, et al: The Wnt5A/protein kinase $\mathrm{C}$ pathway mediates motility in melanoma cells via the inhibition of metastasis suppressors and initiation of an epithelial to mesenchymal transition. J Biol Chem 282: 17259-17271, 2007.

20. Park JI, Venteicher AS, Hong JY, et al: Telomerase modulates Wnt signalling by association with target gene chromatin. Nature 460: 66-72, 2009.

21. Jemal A, Siegel R, Ward E, Hao Y, Xu J, Murray T and Thun MJ: Cancer statistics, 2008. CA Cancer J Clin 58: 71-96, 2008.

22. Thiery JP, Acloque H, Huang RY and Nieto MA: Epithelialmesenchymal transitions in development and disease. Cell 139: 871-890, 2009.

23. Prieur A and Peeper DS: Cellular senescence in vivo: A barrier to tumorigenesis. Curr Opin Cell Biol 20: 150-155, 2008.

24. Pelosi G, Schianchi E, Dell'orto P, Veronesi G, Spaggiari L, Pasini F, Sozzi G, Brambilla E, Griso C and Viale G: Detecting cell-free circulating hTERT mRNA in the plasma may identify a subset of nonsmall cell lung cancer patients. Virchows Arch 448: 7-15, 2006.

25. Gigek CO, Leal MF, Silva PNO, Lisboa LC, Lima EM, Calcagno DQ, Assumpção PP, Burbano RR and Smith MA: hTERT methylation and expression in gastric cancer. Biomarkers 14: 630-636, 2009.

26. Saini N, Srinivasan R, Chawla Y, Sharma S, Chakraborti A and Rajwanshi A: Telomerase activity, telomere length and human telomerase reverse transcriptase expression in hepatocellular carcinoma is independent of hepatitis virus status. Liver Int 29: 1162-1170, 2009.

27. Divella R, Tommasi S, Lacalamita R, et al: Circulating hTERT DNA in early breast cancer. Anticancer Res 29: 2845-2849, 2009.

28. Capkova L, Kalinova M, Krskova L, Kodetova D, Petrik F, Trefny M, Musil J and Kodet R: Loss of heterozygosity and human telomerase reverse transcriptase (hTERT) expression in bronchial mucosa of heavy smokers. Cancer 109: 2299-2307, 2007.

29. Pucciarelli S, Rampazzo E, Briarava M, et al: Telomere-specific reverse transcriptase (hTERT) and cell-free RNA in plasma as predictors of pathologic tumor response in rectal cancer patients receiving neoadjuvant chemoradiotherapy. Ann Surg Oncol 19: 3089-3096, 2012. 
30. Sasaki T, Kuniyasu H, Luo Y, Kitayoshi M, Tanabe E, Kato D, Shinya S, Fujii K, Ohmori H and Yamashita Y: AKT activation and telomerase reverse transcriptase expression are concurrently associated with prognosis of gastric cancer. Pathobiology 81: 36-41, 2014.

31. Chang L, Graham PH, Hao J, Ni J, Bucci J, Cozzi PJ, Kearsley JH and $\mathrm{Li}$ Y: Acquisition of epithelial-mesenchymal transition and cancer stem cell phenotypes is associated with activation of the $\mathrm{PI} 3 \mathrm{~K} / \mathrm{Akt} / \mathrm{mTOR}$ pathway in prostate cancer radioresistance. Cell Death Dis 4: e875, 2013.

32. Li NY, Weber CE, Wai PY, Cuevas BD, Zhang J, Kuo PC and Mi Z: An MAPK-dependent pathway induces epithelial-mesenchymal transition via Twist activation in human breast cancer cell lines. Surgery 154: 404-410, 2013.

33. Kypta RM and Waxman J: Wnt/beta-catenin signalling in prostate cancer. Nat Rev Urol 9: 418-428, 2012.
34. Kahlert UD, Maciaczyk D, Doostkam S, et al: Activation of canonical WNT/ $\beta$-catenin signaling enhances in vitro motility of glioblastoma cells by activation of ZEB1 and other activators of epithelial-to-mesenchymal transition. Cancer Lett 325: 42-53, 2012.

35. Bertrand FE, McCubrey JA, Angus CW, Nutter JM and Sigounas G: NOTCH and PTEN in prostate cancer. Adv Biol Regul 56: 51-65, 2014

36. Cichon MA and Radisky DC: ROS-induced epithelial-mesenchymal transition in mammary epithelial cells is mediated by $\mathrm{NF}-\kappa \mathrm{B}-d e p e n d e n t$ activation of Snail. Oncotarget 5: 2827-2838, 2014.

37. Hoffmeyer K, Raggioli A, Rudloff S, Anton R, Hierholzer A, Del Valle I, Hein K, Vogt R and Kemler R: Wnt/ $\beta$-catenin signaling regulates telomerase in stem cells and cancer cells. Science 336: 1549-1554, 2012. 